\title{
FORMULATION OF A STOCHASTIC SEMI ANALYTICAL FINITE ELEMENT METHOD FOR UNCERTAIN MEDIA
}

\author{
Faker BOUCHOUCHA ${ }^{1}$, Mohamed Najib ICHCHOU ${ }^{2}$, and Mohamed HADDAR ${ }^{1}$ \\ ${ }^{1}$ Unit of Dynamics of the Mechanical Systems (UDSM) \\ National School of Engineers of Sfax. BP. W3038 Sfax -Tunisia \\ e-mail: fakersbouchoucha@yahoo.fr \\ e-mail: mohamed.haddar@enis.rnu.tn \\ ${ }^{2}$ Laboratory of Tribology and Dynamics of Systems (LTDS) \\ Ecole Centrale de Lyon. 36 Avenue Guy de Collongues 69130 Ecully. France \\ Mohamed.Ichchou@ec-lyon.fr
}

Keywords: Stochastic, semi analytical method, finite element, uncertain media, dispersion.

Abstract. In the present work, the stochastic semi analytical finite element (S.S.A.F.E) method for uncertain media is formulated. This method leads to the characterisation of the guided wave propagation through the classical linearization of the governing equation of motion. In probabilistic methods, uncertainty in the parameters is considered and is represented by a random variable. The statistics of the propagation constants and the wave modes are offered. The current study extends the S.A.F.E. techniques to stochastic media through the second order perturbation. A parametric approach for uncertainties treatments is considered and combined to the S.A.F.E. technique. The originality of this paper is the study of the second order perturbation. The sensitivity and the precision of the S.S.A.F.E. approach is treated through the second order perturbation introduced in the structural parameters. The question of the statistics of wave characteristics is considered. The second order perturbation of the propagation constants is investigated. The numerical accuracy and the computational efficiency of the method are demonstrated by comparison with analytical results. Comparisons between numerical results and Monte Carlo simulations of the second order stochastic formulation are among the offered originalities of this work. 


\section{INTRODUCTION}

Guided waves are still a subject of intensive research such as structural forms occur in several engineering areas. This research focuses on the study of guided wave properties and applications. To study wave propagation in structural waveguides, semi-analytical finite element (SAFE) method was investigated by many researchers. Recently, Hayashi and Kawashima [1] derived the SAFE formulation through virtual work principles and proposed a way to calculate the group velocity using the eigensolution at a given frequency. Damljanovic and Weaver [2] developed the linear triangular elements for SAFE method using Lagrange's equations to investigate the elastic waves in waveguides of arbitrary cross-section. Gavric [3] calculated the dispersion relationship in a free rail by using triangular and quadrilateral elements, with those elements obtained from Hamilton's principle. The SAFE method has also be adopted to investigate the wave propagation characteristics for thin-walled structures in [4], where the polynomial interpolation is used in the propagation axis thus leads to the polynomial eigenvalue problems. The method is extended to curved structures in [5], where isoparametric element is used. In the current work, we introduce the semi-analytical numerical method that may be used for wave propagation and dynamic analysis of waveguide structures.

In the literature, however, most of founded numerical issues of wave propagation simulations are mainly limited to deterministic media. In this context the concept of a random field can't be ignored. The nature of variability of the material properties in the structure created a variety of structural problems, in which the uncertainties in different parameters play a major part. Uncertainties can be due to the lack of good knowledge of material properties or due to the change in the load and support condition. The modelling technique is also one of the major sources of uncertainty, in the analysis of waveguides. Hence, when the variations are large, the probabilistic models are advantageous than the deterministic ones. In probabilistic methods, uncertainty in the parameters is considered and is represented by a random variable or field. Among the different probabilistic methods in mechanics, direct Monte Carlo simulation, which involves sampling and estimation, is an example for a frequently used statistical approach $[6,7,8]$. There are also many no statistical methods in probabilistic mechanics. Random perturbation method, orthogonal polynomials expansion methods, Neumann expansion, and numerical integration come under the category of no statistical schemes [9]. Ajith and Gopalakrishnan [10] presented a paper to study the wave propagation responses at high frequencies in composite structures in an uncertain environment using Neumann expansion under the environment of spectral finite element.

The main contribution in the present work is the formulation of the stochastic semi analytical finite element (SSAFE) method through the second order perturbation. We introduce an uncertainty in the structural parameters and using the probabilistic tools, we study the perturbation which affects the wave propagation in the structural waveguide. The numerical accuracy and the computational efficiency of this method are demonstrated by comparison with Monte Carlo simulation and analytical results.

This paper is organised as follows: the next section describes the SAFE method based on the virtual work principle. This method leads to the characterisation of the guided wave propagation through the classical linearization of the governing equation of motion. In the third section, the probabilistic phenomenon is introduced and the SSAFE is formulated using the second order perturbation. Numerical results and discussion are presented in the section 4 . The numerical efficiency of this method is demonstrated by comparison with MC simulations and analytical results. A conclusion together with a description of the work in progress is ultimately given. 


\section{DESCRIPTION OF THE SEMI ANALYTICAL FINITE ELEMENT (SAFE) METHOD}

In this section, we introduce the semi-analytical numerical method that may be used for wave propagation and dynamic analysis of waveguide structures. The basic formulations are presented to illustrate the merits and shortcomings of the method. In the following subsection, we briefly introduce the SAFE method based on the virtual work principle.

Consider a structural waveguide with a uniform cross section. The weak form based on the virtual work principle for the dynamic problem may be written as

$$
W^{e}=\int_{V}\left\langle\varepsilon^{*}\right\rangle\{\sigma\} d v+\int_{V}\left\langle u^{*}\right\rangle \rho\{\ddot{u}\} d v=0
$$

Where $W^{e}$ is the virtual work of the internal forces, $\rho$ is the density, ${ }^{*}$ denotes virtual quantities, $\varepsilon=\left\{\begin{array}{llllll}\varepsilon_{x x} & \varepsilon_{y y} & \varepsilon_{z z} & 2 \varepsilon_{x y} & 2 \varepsilon_{y z} & 2 \varepsilon_{x z}\end{array}\right\}^{T}$ is the strain vector, $u=\left\{\begin{array}{lll}u_{x} & u_{y} & u_{z}\end{array}\right\}^{T}$ is the displacement field, $\sigma=\left\{\begin{array}{llllll}\sigma_{x x} & \sigma_{y y} & \sigma_{z z} & \sigma_{x y} & \sigma_{y z} & \sigma_{x z}\end{array}\right\}^{T}$ is the stress vector. The virtual wok principle can be formulated as:

$$
W^{e}\left(u^{*}, u\right)=W_{i n t}-W_{e x t}
$$

Where $W_{\text {int }}=\int_{V}\left\langle\varepsilon^{*}\right\rangle\{\sigma\} d v$ and $W_{\text {ext }}=-\int_{V}\left\langle u^{*}\right\rangle \rho\{\ddot{u}\} d v$ are the internal and external virtual work, respectively.

The harmonic waves in a uniformly cross-sectioned waveguide are described by the orthogonal function $\exp (j \omega t-j k x) . k$ is the wave number in $x$ direction, $\omega$ is the circular frequency. The displacement function can be $u(x, y, z, t)=u(y, z) \exp (j \omega t-j k x)$, where $u(y, z)$ describe the amplitudes of the displacements of the waveguide cross-section. Thus the straindisplacement relationship $\{\varepsilon\}=[\mathbf{D}]\{u\}$ becomes

$$
\{\varepsilon\}=\left[\begin{array}{ccc}
\partial / \partial \mathbf{x} & 0 & 0 \\
0 & \partial / \partial \mathbf{y} & 0 \\
0 & 0 & \partial / \partial \mathbf{z} \\
\partial / \partial \mathbf{y} & \partial / \partial \mathbf{x} & 0 \\
0 & \partial / \partial \mathbf{z} & \partial / \partial \mathbf{y} \\
\partial / \partial \mathbf{z} & 0 & \partial / \partial \mathbf{x}
\end{array}\right]\{u\}
$$

The strain-displacement relationship can be written in the following form:

$$
\{\varepsilon\}=\left[\mathbf{D}_{0}+k \mathbf{D}_{1}\right]\{u\}
$$

Where

$$
\mathrm{D}_{0}=\left[\begin{array}{ccc}
0 & 0 & 0 \\
0 & \partial / \partial \mathrm{y} & 0 \\
0 & 0 & \partial / \partial \mathbf{z} \\
\partial / \partial \mathrm{y} & 0 & 0 \\
0 & \partial / \partial \mathbf{z} & \partial / \partial \mathrm{y} \\
\partial / \partial \mathbf{z} & 0 & 0
\end{array}\right], \mathrm{D}_{1}=\left[\begin{array}{ccc}
-\mathrm{j} & 0 & 0 \\
0 & 0 & 0 \\
0 & 0 & 0 \\
0 & -\mathrm{j} & 0 \\
0 & 0 & 0 \\
0 & 0 & -j
\end{array}\right]
$$

The strain-displacement relationship can be written as: 


$$
\{\varepsilon\}=[\mathbf{D}][\mathbf{N}]\left\{u_{i}\right\}
$$

Where $u_{i}=\left\{\begin{array}{lll}u_{x_{i}} & u_{y_{i}} & u_{z_{i}}\end{array}\right\}^{T}$ is the displacement vector of the finite element, [N] is the matrix of the element shape functions.

In the other hand, the relationship between the strain and the stress vectors can be given in the following manner:

$$
\{\sigma\}=[\mathbf{C}]\{\varepsilon\}
$$

Where $[\mathbf{C}]$ is the material stiffness matrix which can be written as:

$$
[C]=\frac{E}{(1+v)(1-2 v)}\left(\begin{array}{cccccc}
1-v & v & v & 0 & 0 & 0 \\
& 1-v & v & 0 & 0 & 0 \\
& & 1-v & 0 & 0 & 0 \\
& & & 1-2 v / 2 & 0 & 0 \\
& & & & 1-2 v / 2 & 0 \\
\text { sym } & & & & 1-2 v / 2
\end{array}\right)
$$

Where $E$ is the modulus of elasticity and $v$ is the poisson ration.

The external virtual work can be developed as follows:

$$
W_{e x t}=\left\langle u_{i}^{*}\right\rangle\left[\mathbf{M}^{\mathbf{e}}\right]\left\{\ddot{u}_{i}\right\}
$$

Where $\left[\mathbf{M}^{\mathbf{e}}\right]$ is the mass matrix which can be given as:

$$
\left[\mathbf{M}^{\mathbf{e}}\right]=\int_{\Omega^{e}} \rho[\mathbf{N}]^{\mathbf{T}}[\mathbf{N}] d \Omega^{e}
$$

Where $\Omega^{e}$ denotes the element domain.

The internal virtual work can be developed as:

$$
W_{\text {int }}=\left\langle q_{i}^{*}\right\rangle\left[\mathbf{K}^{\mathbf{e}}\right]\left\{q_{i}\right\}
$$

Where $\left[\mathbf{K}^{\mathbf{e}}\right]$ is the stiffness matrix which can be written as:

$$
\left[\mathbf{K}^{\mathbf{e}}\right]=\int_{\Omega^{e}}[\mathbf{N}]^{\mathrm{T}}[\mathbf{D}]^{\mathrm{T}}[\mathbf{C}][\mathbf{D}][\mathbf{N}] d \Omega^{e}
$$

Introducing equation (4) in equation (12) leads to:

$$
\left[\mathbf{K}^{\mathrm{e}}\right]=\int_{\Omega^{e}}[\mathbf{N}]^{\mathrm{T}}\left[\mathbf{D}_{\mathbf{0}}+k \mathbf{D}_{1}\right]^{\mathrm{T}}[\mathbf{C}]\left[\mathbf{D}_{\mathbf{0}}+k \mathbf{D}_{1}\right][\mathbf{N}] d \Omega^{e}
$$

Then

$$
\left[\mathbf{K}^{\mathbf{e}}\right]=\left[\mathbf{K}_{\mathbf{0}}^{\mathbf{e}}\right]+k\left[\mathbf{K}_{\mathbf{1}}^{\mathbf{e}}\right]+k^{2}\left[\mathbf{K}_{\mathbf{2}}^{\mathbf{e}}\right]
$$

Where

$$
\begin{gathered}
\mathbf{K}_{\mathbf{0}}^{\mathrm{e}}=\int_{\Omega^{e}} \mathbf{N}^{\mathrm{T}} \mathbf{D}_{\mathbf{0}}{ }^{\mathrm{T}} \mathbf{C} \mathbf{D}_{\mathbf{0}} \mathbf{N} d \Omega^{e} \\
\mathbf{K}_{\mathbf{1}}^{\mathrm{e}}=\int_{\Omega^{e}} \mathbf{N}^{\mathrm{T}} \mathbf{D}_{\mathbf{1}}^{\mathrm{T}} \mathbf{C D}_{\mathbf{0}} \mathbf{N} d \Omega^{e}+\int_{\Omega^{e}} \mathbf{N}^{\mathrm{T}} \mathbf{D}_{\mathbf{0}}{ }^{\mathrm{T}} \mathbf{C} \mathbf{D}_{\mathbf{1}} \mathbf{N} d \Omega^{e} \\
\mathbf{K}_{\mathbf{2}}^{\mathrm{e}}=\int_{\Omega^{e}} \mathbf{N}^{\mathrm{T}} \mathbf{D}_{\mathbf{1}}{ }^{\mathrm{T}} \mathbf{C} \mathbf{D}_{\mathbf{1}} \mathbf{N} d \Omega^{e}
\end{gathered}
$$

Assembly of the element matrices and vectors leads to the governing equation of motion of the waveguide: 


$$
\left[\mathbf{K}_{\mathbf{0}}-\omega^{2} \mathbf{M}+k \mathbf{K}_{\mathbf{1}}+k^{2} \mathbf{K}_{\mathbf{2}}\right] \varphi=\mathbf{0}
$$

Where $\varphi$ denotes the nodal displacement vector.

$\varphi_{1}$ and $\varphi_{2}$ denote the eigenvectors associated to $k$ and $(-k)$, from the equation of motion, we can write:

$$
\left[\mathbf{K}_{\mathbf{0}}-\omega^{2} \mathbf{M}+k \mathbf{K}_{\mathbf{1}}+k^{2} \mathbf{K}_{\mathbf{2}}\right] \varphi_{1}=\mathbf{0}
$$

and

$$
\left[\mathbf{K}_{\mathbf{0}}-\omega^{2} \mathbf{M}-k \mathbf{K}_{\mathbf{1}}+k^{2} \mathbf{K}_{\mathbf{2}}\right] \varphi_{2}=\mathbf{0}
$$

The linearization of the equation of motion can be given in the following form:

$$
\left[\begin{array}{cc}
\mathbf{K}_{1} & \mathbf{K}_{\mathbf{0}}-\omega^{2} \mathbf{M} \\
\mathbf{K}_{\mathbf{0}}-\omega^{2} \mathbf{M} & \mathbf{0}
\end{array}\right]\left\{\begin{array}{c}
k \phi_{1} \\
\phi_{2}
\end{array}\right\}+k^{2}\left[\begin{array}{cc}
\mathbf{0} & \mathbf{K}_{2} \\
\mathbf{K}_{2} & \mathbf{K}_{1}
\end{array}\right]\left\{\begin{array}{c}
k \phi_{1} \\
\phi_{2}
\end{array}\right\}=0
$$

Where $\phi_{1}=\varphi_{1}+\varphi_{2}$ and $\phi_{2}=\varphi_{1}-\varphi_{2}$

The linearized equation of motion presents the eigenvalue problem of the system.

$$
(\mathbf{A}-\lambda \mathbf{B}) \phi=\mathbf{0}
$$

Where $\mathbf{A}=\left[\begin{array}{cc}\mathbf{K}_{\mathbf{1}} & \mathbf{K}_{\mathbf{0}}-\omega^{2} \mathbf{M} \\ \mathbf{K}_{\mathbf{0}}-\omega^{2} \mathbf{M} & \mathbf{0}\end{array}\right], \mathbf{B}=-\left[\begin{array}{cc}\mathbf{0} & \mathbf{K}_{2} \\ \mathbf{K}_{2} & \mathbf{K}_{1}\end{array}\right], \phi=\left\{\begin{array}{c}k \phi_{1} \\ \phi_{2}\end{array}\right\}$ and $\lambda=k^{2}$

The resolution of this eigenvalue problem leads to calculate the wave number and provide the dispersion curves.

\section{FORMULATION OF THE STOCHASTIC SEMI ANALYTICAL FINITE ELEMENT METHOD}

In this section, we formulate the stochastic semi analytical finite element method (SSAFEM). We introduce an uncertainty in the structural parameters and using the probabilistic tools, we study the perturbation which affects the wave propagation in the structure. The stochastic eigenvalue problem is given in the following form:

$$
(\tilde{\mathbf{A}}-\tilde{\lambda} \tilde{\mathbf{B}}) \tilde{\phi}=0
$$

The random variables are modelled by Gaussian variables through a second order perturbation, mathematically: $\tilde{v}=\bar{v}+v_{1} \varepsilon+v_{2} \varepsilon^{2}$ where $\tilde{v}$ is the random variable, $\bar{v}$ its mean, $v_{1}$ is the first order perturbation, $v_{2}$ is the second order perturbation and $\varepsilon$ is Gaussian variable centred and reduced. The polynomial chaos $\left(1, \varepsilon, \varepsilon^{2}\right)$ is used as a supplementary dimension of the problem. Using the polynomial chaos projection of these variables, we can extract their means (deterministic quantity), their first and second order perturbation. The second order development of stochastic variables is adopted, such that:

$$
\tilde{\mathbf{A}}=\overline{\mathbf{A}}+\mathbf{A}_{1} \varepsilon+\mathbf{A}_{2} \varepsilon^{2}, \tilde{\mathbf{B}}=\overline{\mathbf{B}}+\mathbf{B}_{1} \varepsilon+\mathbf{B}_{2} \varepsilon^{2}, \tilde{\lambda}=\bar{\lambda}+\lambda_{1} \varepsilon+\lambda_{2} \varepsilon^{2}, \tilde{\phi}=\bar{\phi}+\phi_{1} \varepsilon+\phi_{2} \varepsilon^{2}
$$

The eigenvalue equation can be written as a deterministic part and a perturbation part. The deterministic part is given as:

$$
(\overline{\mathbf{A}}-\bar{\lambda} \overline{\mathbf{B}}) \bar{\phi}=0
$$

The resolution of the equation (25) leads to calculate the deterministic modes $\bar{\lambda}_{i}$ and $\bar{\phi}_{i}$ The perturbation part leads to calculate the first order perturbation of the eigenmode and is given as: 


$$
(\overline{\mathbf{A}}-\bar{\lambda} \overline{\mathbf{B}}) \phi_{1}+\left(\mathbf{A}_{1}-\lambda \mathbf{B}_{1}-\lambda_{1} \mathbf{B}\right) \phi=0
$$

Multiplying this equation by $\bar{\phi}_{i}^{t}$ gives:

$$
\bar{\phi}_{i}^{t}(\overline{\mathbf{A}}-\bar{\lambda} \overline{\mathbf{B}}) \phi_{1}+\bar{\phi}_{i}^{t}\left(\mathbf{A}_{1}-\bar{\lambda} \mathbf{B}_{1}-\lambda_{1} \overline{\mathbf{B}}\right) \bar{\phi}_{i}=0
$$

The transpose of the deterministic eigenvalue problem gives:

$$
\bar{\phi}_{i}^{t}\left(\overline{\mathbf{A}}^{\mathbf{t}}-\bar{\lambda}_{i} \overline{\mathbf{B}}^{\mathrm{t}}\right)=\mathbf{0}
$$

Using the symmetry of $\overline{\mathbf{A}}$ and $\overline{\mathbf{B}}$, we have

$$
\phi_{i}^{t}\left(\overline{\mathbf{A}}-\bar{\lambda}_{i} \overline{\mathbf{B}}\right)=\mathbf{0}
$$

Then, equation (27) can be simplified as follows:

$$
\bar{\phi}_{i}^{t}\left(\mathbf{A}_{1}-\bar{\lambda} \mathbf{B}_{1}-\lambda_{1} \overline{\mathbf{B}}\right) \bar{\phi}_{i}=0
$$

The first order perturbation of the eigenvalue can be extracted according to the following equation:

$$
\lambda_{1}=\frac{\bar{\phi}_{i}^{t}\left(\mathbf{A}_{1}-\bar{\lambda} \mathbf{B}_{1}\right) \bar{\phi}}{\bar{\phi}_{i}^{t} \overline{\mathbf{B}} \bar{\phi}_{i}}
$$

The orthogonality condition of the eigenmode leads to write:

$$
\bar{\phi}_{i}^{t} \overline{\mathbf{B}} \bar{\phi}_{j}=\delta_{i j}
$$

Therefore

$$
\bar{\phi}_{i}^{t} \overline{\mathbf{B}} \bar{\phi}_{i}=1
$$

We can simplify the equation (31) and give the expression of the first order perturbation of the eigenvalue as:

$$
\lambda_{1}=\bar{\phi}_{i}^{t}\left(\mathbf{A}_{1}-\bar{\lambda} \mathbf{B}_{1}\right) \bar{\phi}_{i}
$$

From the definition of first order perturbation of propagation constants, it can be for interest in many applicative engineering cases to consider wave number statistics which can be considered as a random parameter through the second order perturbation:

$$
\tilde{k}=\bar{k}+k_{1} \varepsilon+k_{2} \varepsilon^{2}
$$

Indeed, from the knowledge of the mean and the first order perturbation of the eigenvalue and using the relationship $\tilde{\lambda}=\tilde{k}^{2}$, we can easily express the statistics of $\tilde{k}$ as follows:

$$
\bar{k}= \pm \sqrt{\bar{\lambda}}
$$

Where $\bar{k}$ is the mean of the wave number.

Developing the first order leads to calculate the first order perturbation of the wave number:

$$
k_{1}=\frac{\lambda_{1}}{2 \bar{k}}
$$

The polynomial chaos projection of the equation (23) leads to give the second order perturbation equation as follows:

$$
\left(\mathbf{A}_{2}-\bar{\lambda} \mathbf{B}_{2}-\lambda_{1} \mathbf{B}_{1}-\lambda_{2} \overline{\mathbf{B}}\right) \bar{\phi}+\left(\mathbf{A}_{1}-\lambda \mathbf{B}_{1}-\lambda_{1} \overline{\mathbf{B}}\right) \phi_{1}+(\overline{\mathbf{A}}-\bar{\lambda} \overline{\mathbf{B}}) \phi_{2}=0
$$

Multiplying this equation by $\bar{\phi}_{i}^{t}$ and using the equation (32), we obtain:

$$
\bar{\phi}^{t}\left(\mathbf{A}_{2}-\bar{\lambda} \mathbf{B}_{2}-\lambda_{1} \mathbf{B}_{1}-\lambda_{2} \overline{\mathbf{B}}\right) \bar{\phi}+\bar{\phi}^{t}\left(\mathbf{A}_{1}-\lambda \mathbf{B}_{1}-\lambda_{1} \overline{\mathbf{B}}\right) \phi_{1}=0
$$

The second order perturbation is then easily extracted

$$
\lambda_{2}=\frac{\bar{\phi}^{t}\left(\mathbf{A}_{2}-\bar{\lambda} \mathbf{B}_{2}-\lambda_{1} \mathbf{B}_{1}\right) \bar{\phi}+\bar{\phi}^{t}\left(\mathbf{A}_{1}-\lambda \mathbf{B}_{1}-\lambda_{1} \overline{\mathbf{B}}\right) \phi_{1}}{\bar{\phi}^{t} \overline{\mathbf{B}} \bar{\phi}}
$$


Using equation (33), we can write:

$$
\lambda_{2}=\bar{\phi}^{t}\left(\mathbf{A}_{2}-\bar{\lambda} \mathbf{B}_{2}-\lambda_{1} \mathbf{B}_{1}\right) \bar{\phi}+\bar{\phi}^{t}\left(\mathbf{A}_{1}-\lambda \mathbf{B}_{1}-\lambda_{1} \overline{\mathbf{B}}\right) \phi_{1}
$$

The second order perturbation of the wave number is offered as follows:

$$
k_{2}=\frac{\lambda_{2}-k_{1}^{2}}{2 \bar{k}}
$$

\section{NUMERICAL RESULTS AND DISCUSSION:}

In this section, we present and discuss the numerical results obtained through the SSAFE method. The validation of this method is done using analytical results and MC simulations. We treat many cases of uncertain media in order to prove that the developed technique is general.

The studied structure is illustrated in figure 1 . We treat the cylindrical structure which has the following characteristics: radius $=100 \mathrm{~mm}$, thickness $=1 \mathrm{~mm}$, length: $\mathrm{d}=20 \mathrm{~mm}$, nodes number $=36$ per section and element number $=36$. We use a surface element with 4 nodes that include 6 d.o.f. per node.

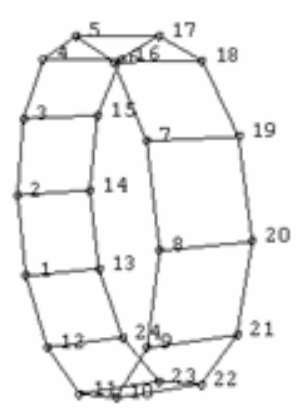

Figure 1: Cylindrical structure.

\subsection{Longitudinal wave's case study}

Figure 2 shows the deterministic dispersion curves for the longitudinal mode. This illustration is obtained from the equation (36): $\bar{k}= \pm \sqrt{\bar{\lambda}}$ where $\bar{\lambda}$ is extracted by solving the deterministic eigenvalue problem $(\overline{\mathbf{A}}-\overline{\lambda \overline{\mathbf{B}}}) \bar{\phi}=0$.

The validation is done using the analytical results and MC simulations. The analytical theory gives the stochastic wave number for the traction compression mode in the following form:

$$
\tilde{k}_{T C}=\omega \sqrt{\frac{\rho}{E}}
$$




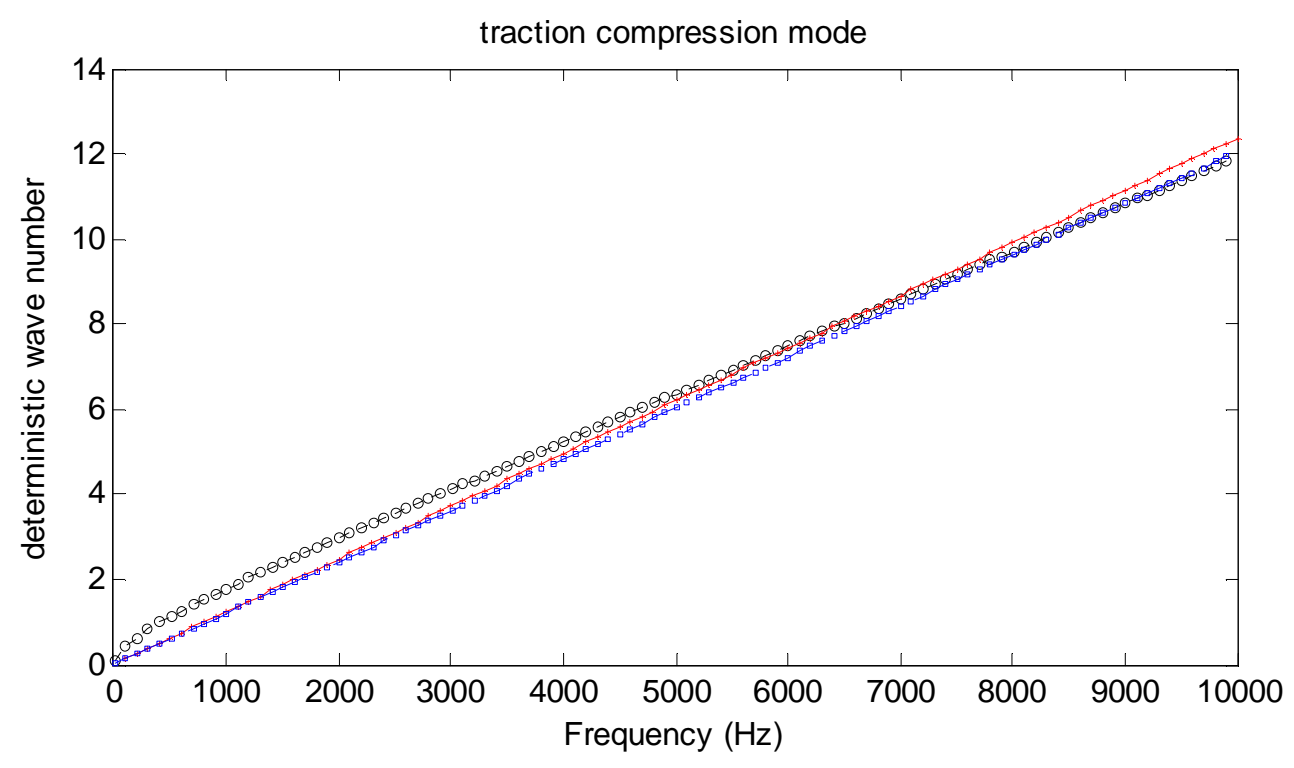

Figure 2: The deterministic wave number for the longitudinal mode

○૦○ SSAFE $\boxminus \boxminus \boxminus$ Analytical $\quad x-X-×$ MC

The Young modulus is an important parameter which affects the stiffness matrix. We introduce an uncertainty in this structural parameter through the second order perturbation and it can be written as a Gaussian distribution: $\tilde{E}=\bar{E}+E_{1} \varepsilon+E_{2} \varepsilon^{2}$ where $\bar{E}=2.1 \times 10^{5} \mathrm{MPa}$, $E_{1}=0.05 \bar{E}$ and $E_{1}=0.05 \bar{E}$

Figure 3 presented the first order perturbation of the wave number for the longitudinal mode following an uncertainty introduced in the Young modulus. The first order perturbation of the wave number is given in equation (37).

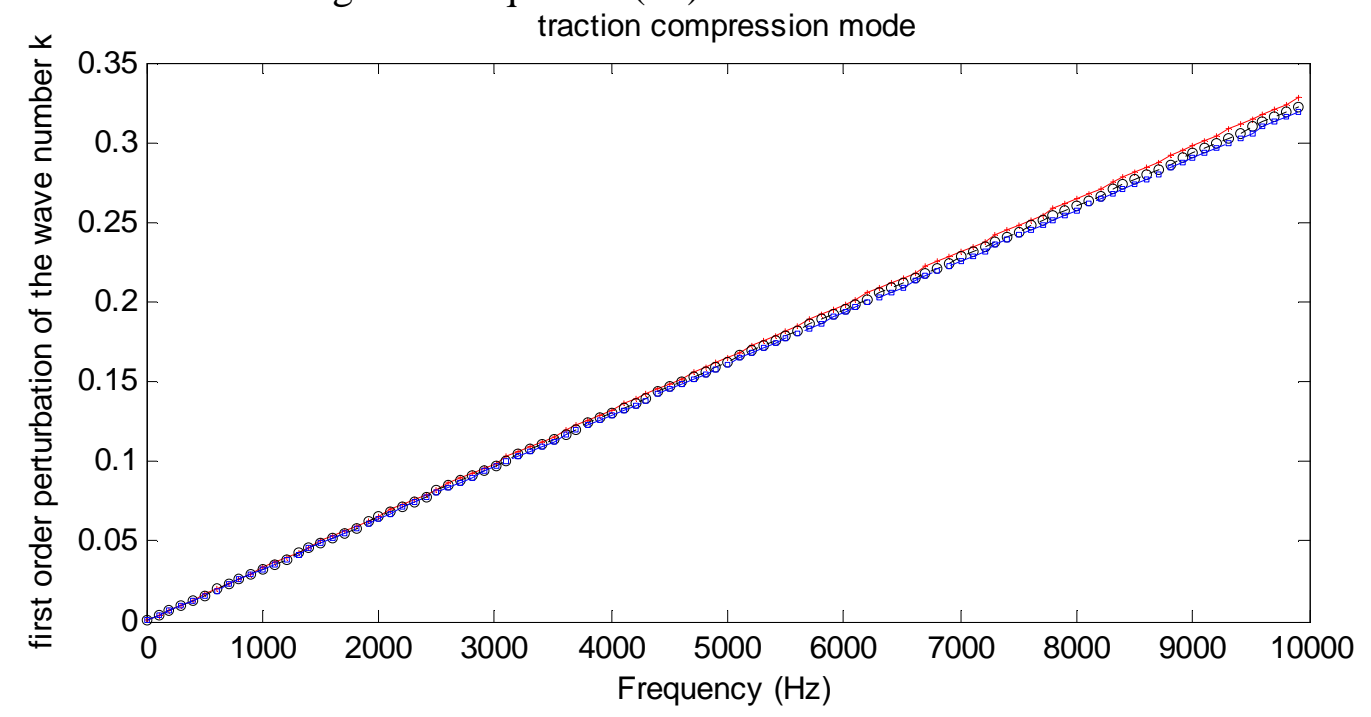

Figure 3: The first order perturbation of the wave number for the longitudinal mode (E stochastic)

$$
\text { ○૦९SSAFE } \boxminus \boxminus \boxminus \text { Analytical } \quad x-X-× \mathrm{MC}
$$

Figure 4 shows the second order perturbation of the wave number (equation 42) for the longitudinal mode through the perturbation introduced in the Young modulus. 


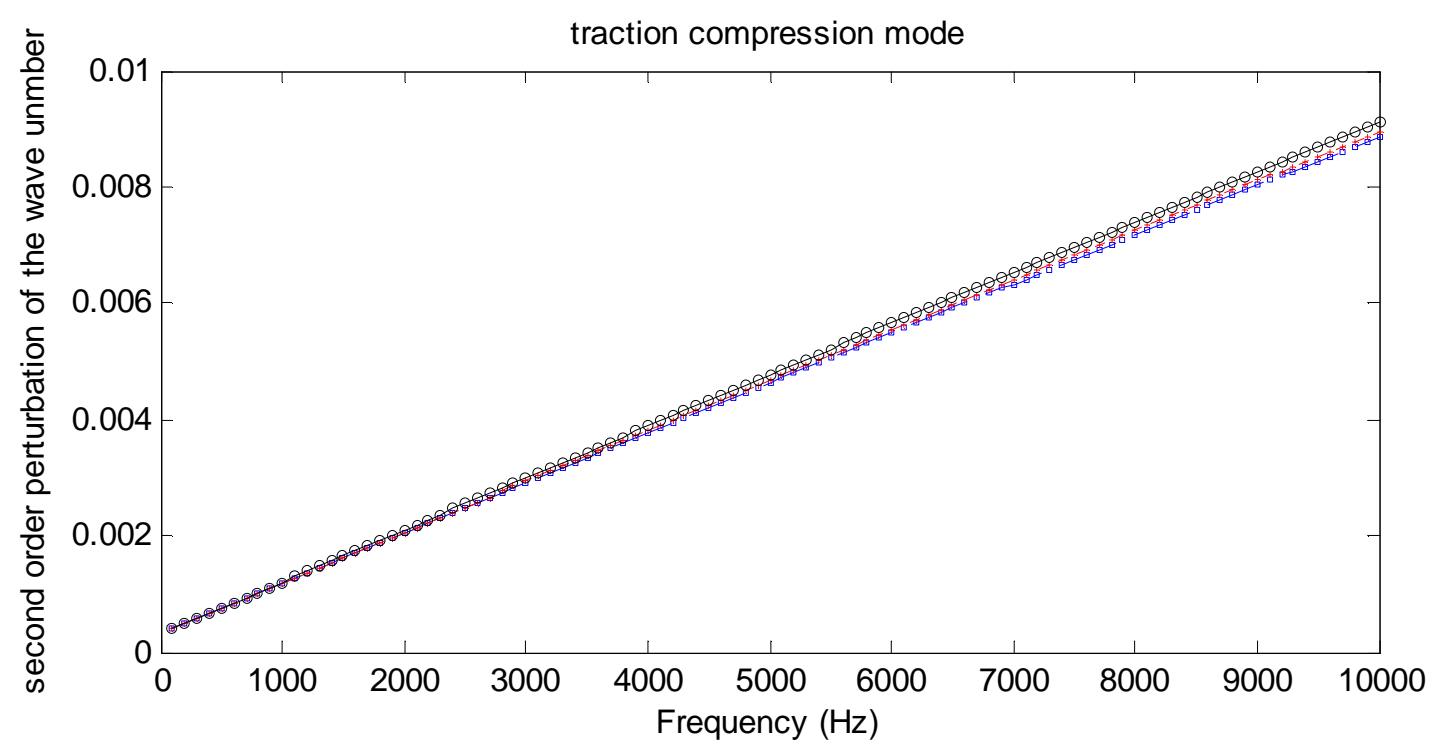

Figure 4: The second order perturbation of the wave number for the longitudinal mode (E stochastic)

$$
\text { ○૦९ SSAFE } \boxminus \boxminus \boxminus \text { Analytical } \quad x-x-x \text { MC }
$$

\subsection{Flexural wave's case study}

For the flexural mode, the deterministic, first order and second order perturbation of the wave number are illustrated in figures 5, 6 and 7, respectively. The analytical validation is done using the analytical equation:

$$
\tilde{k}_{\text {flexion }}=\left(\frac{\rho S \omega^{2}}{E I}\right)^{1 / 4}
$$

Where $E I$ is the bending stiffness $\rho$ is the mass density, $S$ is the cross section and $\omega$ is the pulsation.

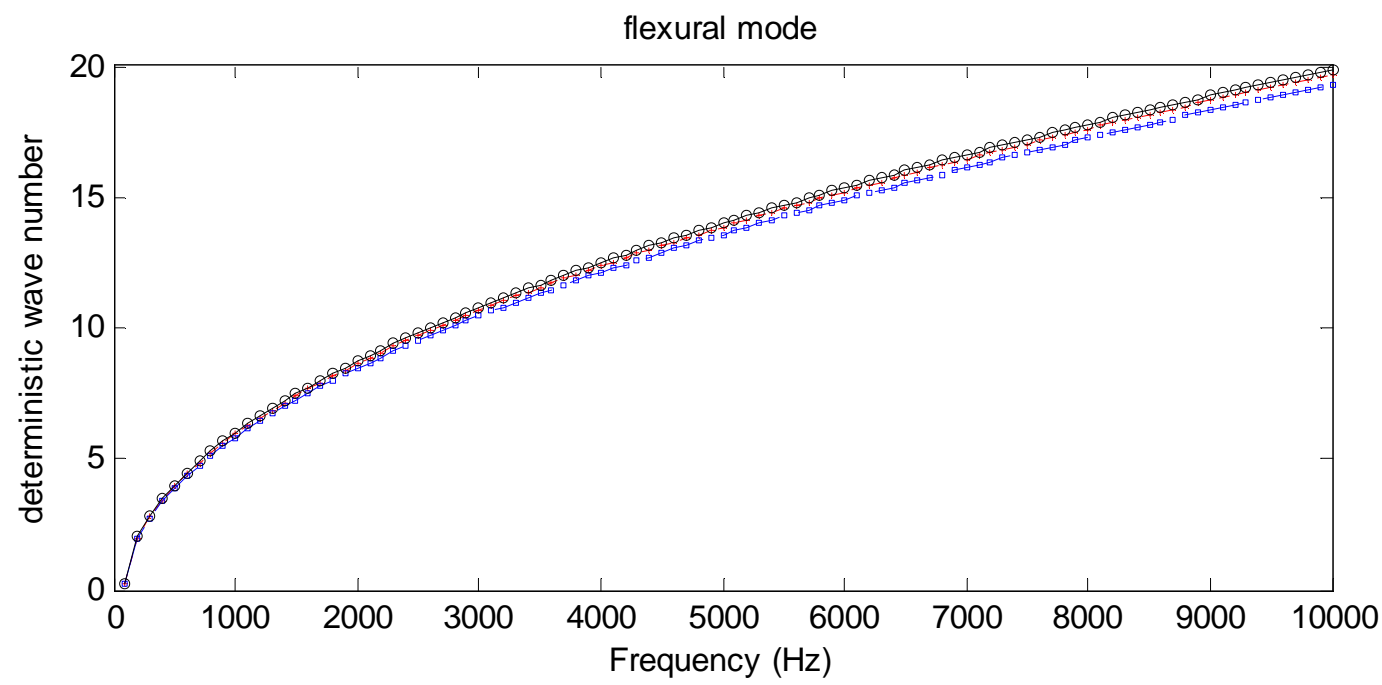

Figure 5: The deterministic wave number for the flexural mode

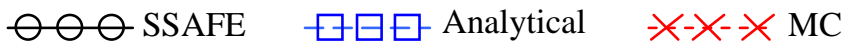




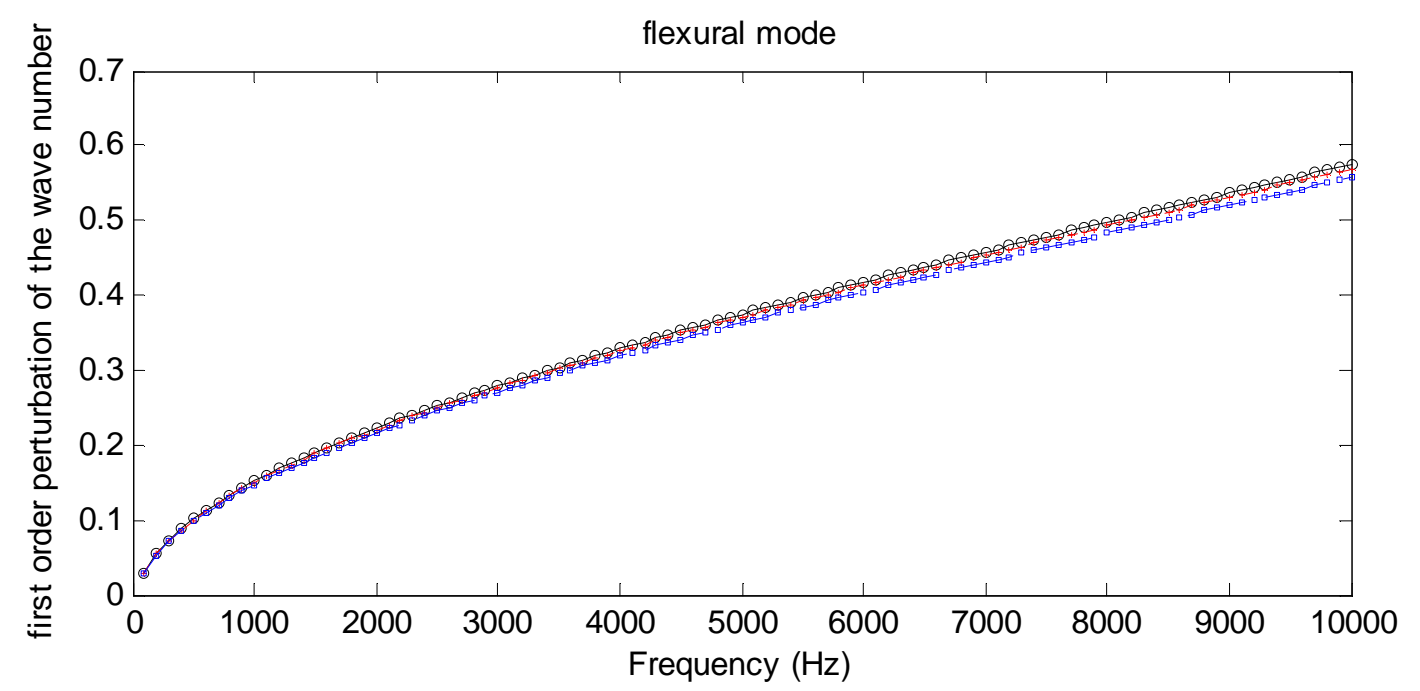

Figure 6: The first order perturbation of the wave number for the flexural mode (E stochastic)

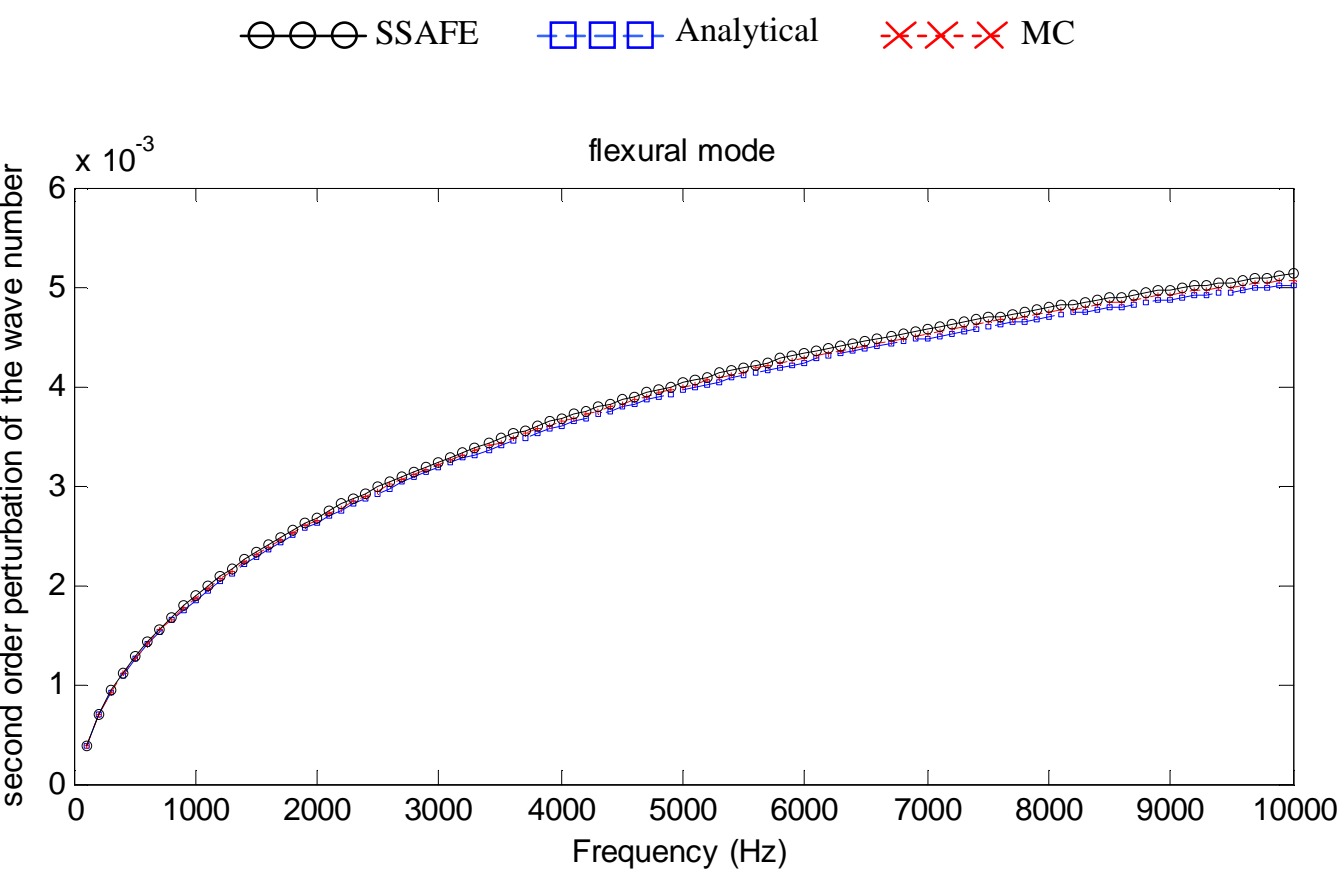

Figure 7: The second order perturbation of the wave number for the flexural mode (E stochastic)

$$
\text { ○૦९ SSAFE } \boxminus \boxminus \boxminus \text { Analytical } \quad x-x-× \text { MC }
$$

\section{CONCLUSION}

In this paper, the issue of wave propagation parameters estimations in random waveguide was dealt with. Guided wave properties are an important physical notion for use in many engineering problems, the paper results are expected to be helpful in non-deterministic problems and a formulation named Stochastic Semi Analytical Finite Element was offered. This formulation allows wave characteristics to be defined by means of a stochastic finite element model through a parametric probabilistic approach. The formulation is general and can be applied to various structural shapes. The uncertainty is introduced in the structural parameters through second-order perturbation and using the probabilistic tools, we study the statistics of the wave's dispersion. These statistics are expected to be of use in the sensitivity analysis of guided waves techniques for wave propagation. Ultimately, numerical and analytical comparisons were given. Such analytical developments and numerical experiments showed the 
effectiveness of the proposed approach to predict the statistics of guided wave propagation characteristics. The main paper finding can be extracted as follows:

1. The stochastic semi analytical finite element (SSAFE) method is formulated through the second order perturbation. The statistics of the dispersion curves are extracted following an uncertainty introduced in the structural parameters and using the probabilistic tools

2. The numerical accuracy and the computational efficiency of this method are demonstrated by comparison with the MC simulations and the analytical results.

The SSAFE offers some interesting research perspectives. Among future work, the use of SSAFE for energy issues in complex wave guide is an important task. Among the investigations in progress, the mid and high frequencies behaviour is the main target in this case. Further investigations are under progress in order to use such numerical methods in the context of smart materials and structures

\section{REFERENCES}

[1] V. Damljanovic , R.L. Weaver, Propagating and evanescent elastic waves in cylindrical waveguides of arbitrary cross section. Journal of the Acoustical Society of America, 115, 1572-1581, 2004.

[2] S. Finnveden, Evaluation of modal density and group velocity by a finite element method. Journal of Sound and Vibration, 273, 51-75, 2004.

[3] S. Finnveden, M. Fraggstedt, Waveguide finite elements for curved structures. Journal of Sound and Vibration, 312, 644-671, 2008.

[4] L. Gavric, Computation of propagative waves in free rail using a finite element technique. Journal of Sound and Vibration, 185, 420-432, 1995.

[5] T. Hayashi, W.J. Song, J.L. Rose. Guided wave dispersion curves for a bar with an arbitrary cross-section, a rod and rail example. Ultrasonics, 41, 175-183, 2003.

[6] F. James, Monte Carlo Theory and Practice. Rep. Prog. Phys, 43 (9), 1145-1189, 1980.

[7] M.K. Decker, The Monte-Carlo Theory in Science and Engineering: Theory and Application. Comput. Methods Appl. Mech. Eng, 89 (1), 463-483, 1991.

[8] G.I. Schueller, Computational Stochastic Mechanics - Recent Advances. Comput. Struc, 79 (22-25), 2225-2234, 2001.

[9] J. Li, J.B. Chen, The Probability Density Evolution Method for Dynamic Response Analysis of Non-Linear Stochastic Structures. Int. J. Numer. Methods Eng, 65 (6), 882903, 2006.

[10] V. Ajith, S. Gopalakrishnan. Spectral Element Approach to Wave Propagation in Uncertain Composite Beam Structures. Journal of Vibration and Acoustics, 133(5), 2011, DOI: $10.1115 / 1.4003945$. 